\title{
Das Establishment schlägt zurück? Die Präsidentschaftsvorwahlen der Republikaner im Jahr 2012
}

\author{
Robert Matschoß und Torben Lütjen
}

Eigentlich hätte es 2012 ein von Optimismus getragener kraftvoller Vorwahlkampf für die Republikaner werden müssen. Schließlich hatten sie bei den Midterm Elections zwei Jahre zuvor gerade die Mehrheit im US-Repräsentantenhaus von den Demokraten zurückerobert - und das mit dem klarsten Sieg seit 1948. „A shellacking“, eine Abreibung, hatte Präsident Barack Obama diese Niederlage genannt, und in den Wochen danach schien eine euphorisierte Republikanische Partei vor lauter Kraft kaum noch laufen zu können. Das nächste Ziel für sie war also klar: der Sieg bei den Präsidentschaftswahlen 2012. Und obgleich sich die wirtschaftliche Lage seit 2010 ein wenig gebessert hatte, waren die Ausgangsbedingungen für den Demokratischen Amtsinhaber noch immer nicht gerade rosig.

Doch wie es bisweilen mit plötzlichen und spektakulären Mobilisierungsschüben in Parteien ist: manchmal wird durch sie die Schraube auch ein Stück zu weit gedreht, weil sie eine Dynamik entfalten, die zwar die eigene Basis immer weiter motiviert - durch die aber gleichzeitig die Partei in eine Richtung gesteuert wird, die in der Außenwahrnehmung nicht unbedingt vorteilhaft ist. Vor dem Hintergrund des konservativen Backlash, den Amerika seit Anfang von Obamas erster Amtszeit erlebt hatte und der in der Entstehung der Tea Party seine deutlichste Ausprägung gefunden hatte, erschien die Annahme plausibel, die Republikaner könnten einen Vertreter des radikal-konservativen Flügels zum Kandidaten küren. Ein spannendes Experiment wäre das gewesen, denn es hätte die Probe aufs Exempel gemacht, wie mehrheitsfähig der amerikanische Ultra-Konservativismus in der US-Gesellschaft wirklich ist.

Doch ganz so kam es nicht: Nominiert wurde schließlich der als vergleichsweise moderat geltende Mitt Romney, der die Republikanischen Vorwahlen klar für sich entscheiden konnte. Mit ihm gewann der Kandidat, dem von vornherein die größten Siegchancen prognostiziert worden waren. Er war 2011 der erfolgreichste Spendensammler, verfügte über einen landesweit hohen Bekanntheitsgrad, der vor allem von seiner Kampagne 2008 herrührte, erhielt ein hohes Maß an Medienaufmerksamkeit, und zum Jahreswechsel 2011/2012 hatten sich bereits weit mehr Angehörige des Republikanischen Establishments für ihn ausgesprochen (so genannte Endorsements) als für seine Mitbewerber. ${ }^{1}$

Hat also das Establishment gesiegt und die radikaleren Teile der eigenen Wählerkoalition eindämmen können? Oder haben sich die konservativen Strömungen innerhalb des Republikanischen Spektrums gegenseitig bekämpft und so dem Kandidaten der moderaten innerparteilichen Minderheit zum Sieg verholfen? Aber auch das ist eine Möglichkeit: Vielleicht war selbst Romneys Sieg am Ende doch ein Erfolg der Tea Party, weil der „Front-Runner“, der Spitzenreiter, ihnen programmatisch so weit entgegen kommen musste, dass dadurch der Standpunkt der Partei abermals nach rechts verschoben wurde.

1 Vgl. John Sides / Lynn Vavreck, Random, or Romney?, in: dies., The Gamble, Princeton 2012, S. 6-55, S. 43 ff., Online-Vorabdruck http://press.princeton.edu/chapters/s3_9921.pdf (Abruf am 24. Januar 2013). 


\section{Eine neue Wettbewerbsordnung: Der Aufstieg der Super PACs und die geänderten Regeln des Republican National Committee}

Die Präsidentschaftsvorwahlen in den USA spielen sich stets in einem komplexen Geflecht aus Regeln der nationalen Parteiorganisation, den Gesetzen der Einzelstaaten und den Regeln der dortigen Parteiorganisationen ab. Dabei ist die Geschichte der Präsidentschaftsvorwahlen in den USA auch eine Geschichte kontinuierlicher Reformen. Seitdem sich die Presidential Primary Mitte der 1970er Jahre endgültig als dominantes Nominierungsverfahren durchgesetzt hatte, unternahmen die Parteien stets neue Versuche, den Prozess zu optimieren. Im Gegensatz zu den Demokraten galten die Republikaner dabei als vergleichsweise reformscheu. ${ }^{2}$ Im Einklang mit der Betonung des Subsidiaritätsprinzips im amerikanischen Konservatismus machte das Republican National Committee (RNC) den Einzelstaaten weniger strenge Vorgaben. Die Zurückhaltung bei Reformen liegt auch in den eingeschränkten Kompetenzen des RNC begründet, das - anders als das Democratic National Committee (DNC) - bisher nicht befugt war, die Regeln für die Vorwahlen zwischen den National Conventions zu verändern. Zudem finden diese Nominierungsparteitage nur alle vier Jahre statt. 2008 jedoch, sicherlich auch als Reaktion auf den von vielen in der Partei kritisch beurteilten Verlauf der Vorwahlsaison jenes Jahres, räumte der Parteitag dem RNC die Kompetenz ein, Teile des Vorwahlprozesses eigenständig zu verändern. ${ }^{3}$

Im August 2010 ließ das RNC Taten folgen. Die Republikanischen Präsidentschaftsvorwahlen 2012 fanden gleich in zweierlei Hinsicht unter veränderten Spielregeln statt. Erstens beschränkte das RNC die Freiheit der Einzelstaaten bezüglich der Terminierung: Ursprünglich sollte keine Vorwahl im Januar abgehalten werden. Abgesehen von den vier ersten Staaten (Iowa, New Hampshire, South Carolina und Nevada) hätten zudem alle Vorwahlen nach dem 6. März stattzufinden. Zuwiderhandlungen sollten mit einer Halbierung der Delegiertenzahl auf der National Convention der Partei bestraft werden.

Eine zweite Neuregelung betraf den Verteilungsschlüssel für die Delegiertenstimmen. Bis April 2012 sollten keine so genannten Winner-take-all Vorwahlen stattfinden, bei denen der Sieger alle Stimmen eines Staates erhält und die traditionellerweise bei den Republikanern häufig zur Anwendung kommen. ${ }^{4}$ Die neuen Regeln waren auch eine Reaktion auf das Phänomen des „Frontloading“, das die Entwicklung hin zu einer Häufung der Vorwahlen zu Beginn der Vorwahlsaison beschreibt. ${ }^{5}$ Klares Ziel dieser veränderten Spielregeln war es, das Nominierungsverfahren in die Länge zu ziehen. ${ }^{6}$ Eine Blitzentscheidung wie 2008, als John McCain bereits im Februar de facto als Kandidat feststand, während sich bei den Demokraten Hillary Clinton und der spätere Sieger Barack Obama bis zum Schluss ein span-

2 Vgl. Brian F. Schaffner, Politics, Parties, and Elections in America, Boston 2012, S. 169 ff.

3 Vgl. Rob Richie / Elise Helgesen, A Rogue Convention? How GOP Party Rules May Surprise in 2012, in: Politico vom 25. November 2011, http://www.politico.com/news/stories/1111/69048. html (Abruf am 13. Februar 2013).

4 Eine detaillierte Übersicht über die neuen Regeln ist auf den Seiten von FairVote zu finden, http:// www.fairvote.org/delegate-allocation-rules-in-2012-gop (Abruf am 10. Februar 2013).

5 Für eine detaillierte Analyse siehe William G. Mayer / Andrew E. Busch, The Front-Loading Problem in Presidential Nominations, Washington D.C. 2004.

6 Vgl. Rhodes Cook, 2012 Presidential Nominating Process: Time for The States, http://www.rasmussenreports.com/public_content/political_commentary/commentary_by_rhodes_cook/2012_presidential_nominating_process_it_s_time_for_the_states (Abruf am 13. Februar 2013). 
nendes und mobilisierungsförderndes Rennen um die Kandidatur lieferten, sollte verhindert werden. $^{7}$

Der Grundkonflikt zwischen den Interessen der Einzelstaaten und der Bundespartei wurde jedoch auch damit nicht gelöst: Während letztere daran interessiert ist, genügend Raum zwischen den einzelnen Vorwahlen zu lassen, damit sich Momentum entwickeln kann und die Kandidaten genug Zeit haben, Spenden zu sammeln und Organisationen in weiteren Einzelstaaten aufzubauen, wollen erstere ihren Wählern einen möglichst großen Einfluss auf die Nominierungsentscheidung ermöglichen und daher ihren Vorwahltermin möglichst früh ansetzen. ${ }^{8}$ Fünf Staaten (Arizona, Florida, Michigan, New Hampshire und South Carolina) ließen es auf einen offenen Konflikt ankommen, und die Partei bestrafte sie tatsächlich mit der Halbierung ihrer Delegiertenzahl auf dem Parteitag im August.

Eine weitere Neuerung gegenüber 2008 ergab sich auf dem Gebiet der Wahlkampffinanzierung. Die Präsidentschaftsvorwahlen 2012 waren die ersten, in denen die so genannten Super PACs eine wesentliche Rolle spielten. Diese Organisationen sind in Folge der Rechtsprechung des Supreme Court im Fall Citizens United v. Federal Election Commission und deren Anwendung im Fall SpeechNow.org v. Federal Election Commission entstanden. ${ }^{9}$ Anders als reguläre Political Action Committees (PACs) dürfen sie keine Spenden direkt an einen Kandidaten oder dessen Wahlkampforganisation weitergeben. ${ }^{10}$ Als so genannte „independent expenditure-only committees“ können sie jedoch unabhängig von den Kandidatenorganisationen Spenden in unbegrenzter Höhe von Einzelpersonen, Unternehmen, Gewerkschaften und Verbänden annehmen und auch Summen in unbegrenzter Höhe ausgeben, um direkt für die Wahl oder Abwahl eines Kandidaten zu werben. Sie müssen der Federal Election Commission aber regelmäßig über ihre Spender Bericht erstatten. ${ }^{11}$

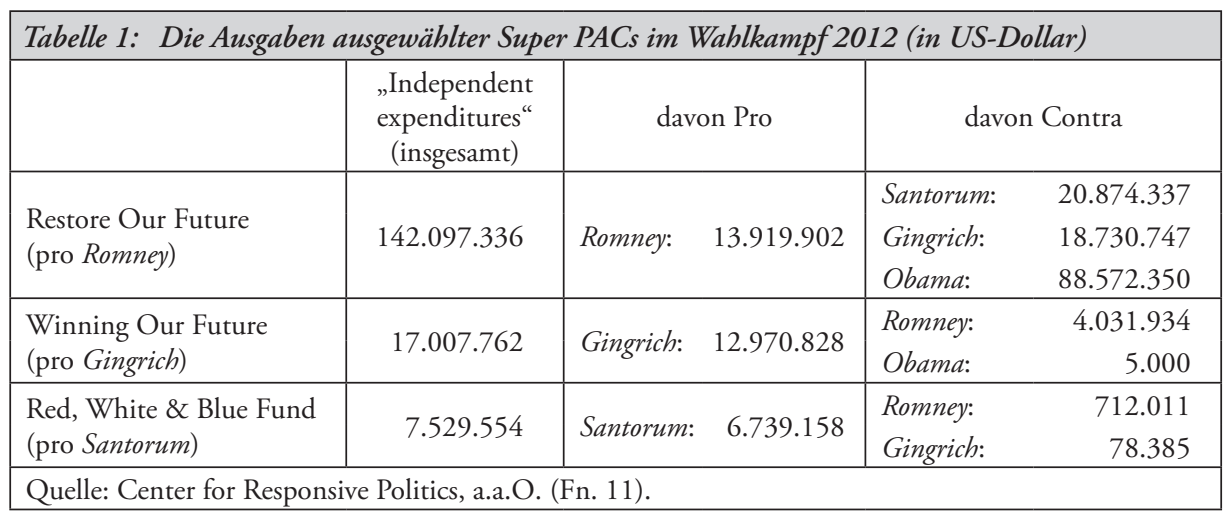

7 Vgl. Rob Richie / Elise Helgesen, a.a.O. (Fn. 3).

8 Vgl. Caroline Tolbert / Peverill Squire, Reforming the Presidential Nomination Process, Editors' Introduction, in: Political Science \& Politics, 42. Jg. (2009), H. 1, S. 27 - 32, S. 31.

9 Vgl. Michael M. Franz, Interest Groups in Electoral Politics, in: The Forum, 10. Jg. (2012), H. 4, S. 62 - 79, S. 63.

10 Jedoch ist die offensichtliche Koordinierung vielfach gar nicht nötig für die Kooperation mit den Parteien. Siehe dazu Maik Bohne, Vibrant Spaces. Dynamik und Struktur von Parteiräumen in US-amerikanischen Wahlkämpfen, Opladen 2011, S. 191 - 220.

11 Vgl. Center for Responsive Politics, Super PACs, http://www.opensecrets.org/pacs/superpacs.php (Abruf am 25. Februar 2013). 
Ihrer Unabhängigkeit von den Kandidaten verdanken die Super PACs auch ihre strategische Flexibilität - besonders die Entscheidung für „negative campaigning “ scheint ihnen leichter zu fallen: Engagiert sich ein Super PAC für einen Kandidaten, indem es dessen Gegner scharf angreift, muss der Kandidat selbst nicht in die Niederungen der Auseinandersetzung hinabsteigen und kann sich weiter untadelig geben. Und so hatten die Super PACs entscheidenden Anteil an dem vergleichsweise negativen Ton der Vorwahlsaison 2012. „Restore Our Future“ aus dem Romney-Lager hat in Iowa beispielsweise von November bis Januar 2,7 Millionen Dollar für Fernsehspots ausgegeben, die Newt Gingrich attackierten. ${ }^{12}$ Bis Mitte Mai 2012 finanzierte dieses Super PAC etwa doppelt so viel Werbung und Direct Mail wie Romneys Wahlkampforganisation selbst, nämlich 44,5 Millionen Dollar. ${ }^{13}$ Insgesamt wurden in der Vorwahlsaison 2012 fast 56 Prozent der gesendeten Wahlwerbespots von Super PACs finanziert. ${ }^{14}$ Tabelle 1 zeigt die Gewichtung bei den Ausgaben des jeweils wichtigsten Super PACs der drei Kandidaten Gingrich, Romney und Santorum. Alle drei verwendeten weit mehr Ressourcen auf die Arbeit gegen die innerparteiliche Konkurrenz (und gegen Obama) als zur Anpreisung desjenigen Kandidaten, hinter dem sie eigentlich standen. Zudem wird die relative Stärke von „Restore Our Future“ deutlich: Allein dieses Super PAC hat etwa dreimal so viel Geld in Kampagnen gegen Santorum investiert, wie der „Red, White \& Blue Fund“ für Santorum ausgegeben hat.

Trotz dieser Unterschiede in ihrer Finanzausstattung boten Super PACs auch Chancen für diejenigen Kandidaten, die selbst keine so finanzkräftige Wahlkampforganisation hatten und vielleicht beim Sammeln von regulären Spenden nicht so erfolgreich waren. So liegt die Vermutung nahe, dass auch das Auftreten der Super PACs zur Länge des Vorwahlkampfes der Republikaner beigetragen hat, indem weniger erfolgreiche Kandidaten länger im Rennen gehalten wurden. ${ }^{15}$

\section{Anybody but Romney? Kandidatenfeld und ideologische Strömungen des Vorwahlkampfes}

Die Vorwahlen sind auch deswegen von Bedeutung, weil sie in besonderer, prägnanter Art und Weise etwas über den Zustand der Parteien aussagen: über ihr Selbstvertrauen und Selbstverständnis, welche Strömungen gerade dominant sind und welche Themen im Vordergrund stehen. Vor allem aber funktionierten die Nominierungsprozesse von Demokraten und Republikanern in letzter Zeit wie Einfallstore, durch die neue politische und soziale Bewegungen ihren Einfluss über eine der beiden Parteien auszudehnen versuchten. ${ }^{16} \mathrm{Da}$ Parteien in den USA nicht im gleichen Maße wie ihre europäischen Gegenstücke als perma-

12 Vgl. Reid Wilson, The Super PAC Arrives, in: National Journal vom 5. Januar 2012, http://www. nationaljournal.com/columns/on-the-trail/the-super-pac-arrives-20120105 (Abruf am 13. Februar 2013).

13 Vgl. Nicholas Confessore, 'Super PACs' Let Strategists Off the Leash, in: New York Times vom 20. Mai 2012, http://www.nytimes.com/2012/05/21/us/politics/super-pacs-changing-how-politicaloperatives-operate.html (Abruf am 13. Februar 2013).

14 Vgl. Michael M. Franz, a.a.O. (Fn. 9), S. 67.

15 Vgl. ebenda, S. 67.

16 Vgl. für einen gerafften Überblick Andrew E. Busch, Political Movements, Presidential Nominations, and the Tea Party, in: William G. Mayer / Jonathan Bernstein (Hrsg.), The Making of the Presidential Candidates 2012, Lanham 2012, S. 59 - 92. 
nente Organisationen existieren, die sich in einem kontinuierlichen Prozess über ihre programmatische Ausrichtung verständigen, sind und waren Kandidatenentscheidungen auch oft ideologische Richtungsentscheidungen, durch die dann fortan der Kurs der Parteien bestimmt wurde.

Insbesondere die jüngere Geschichte der Republikaner ist eine Aneinanderreihung solcher Richtungskämpfe zwischen den Parteiflügeln bei der Nominierung der Präsidentschaftskandidaten. Schon seit den 1950er Jahren - noch bevor sich das System der Vorwahlen flächendeckend in den meisten Bundesstaaten durchgesetzt hatte - gab es Versuche amerikanischer Konservativer, den ideologischen Standpunkt der Republikanischen Partei in ihre Richtung zu verschieben. Die meiste Zeit über siegte dabei der moderate, ja eigentlich schon liberale „Rockefeller-Flügel“. ${ }^{17}$ Nur 1964 gelang es den Anhängern von „limited government“ und hartem Antikommunismus, „ihren Kandidaten“ Barry Goldwater zu nominieren. Dieser sollte zwar in der Hauptwahl mit fliegenden Fahnen gegen den damals noch populären amtierenden Präsidenten Lyndon B. Johnson untergehen. Aber immerhin konnte Goldwater fünf Bundesstaaten im Süden der USA gewinnen - was nicht nur den Anfang vom Ende der Vorherrschaft der Demokraten in dieser Region bedeutete, sondern auch das folgenreichste Realignment der zweiten Hälfte des 20. Jahrhunderts auslöste und die Parteien ideologisch weit voneinander entfernte. ${ }^{18}$ Erst 1980 aber war die konservative Revolution dann vollendet: Mit Hilfe der religiösen Rechten und Jerry Falwells „Moral Majority“ errang Ronald Reagan die Nominierung der Republikaner. Mit der Integration der evangelikalen Wählerschaft hatte sich der ideologische Standort der Partei noch einmal eindeutig nach rechts verschoben. Nach Reagans Nominierung und seinem anschließendem Sieg über Jimmy Carter war die längst brüchig gewordene Herrschaft des liberalen Ostküsten-Establishments endgültig beendet. ${ }^{19}$

In eben diese Tradition des ewigen Konfliktes zwischen dem moderaten und strengkonservativen Flügel der Partei haben nicht wenige Beobachter innerhalb und außerhalb der USA auch die Vorwahlen des Jahres 2012 eingeordnet, denn schließlich tobte in der Grand Old Party, so die gängige Interpretation, ein Kampf zwischen dem Parteiestablishment und den Anhängern der Tea Party - jener radikal-libertären Protestbewegung, die 2009 nur kurz nach Obamas Amtsanritt entstand und in den Midterm Elections 2010 mit ihrer Unterstützung besonders konservativer Kandidaten einige beachtliche Erfolge erzielen konnte. Eine der Schlüsselfragen des Wahlkampfes lautete daher, wie stark der Einfluss der Tea Party auf die Republikanische Partei mittlerweile war und ob es ihr gelingen würde, einen Kandidaten nach ihren Vorstellungen durchzusetzen.

Die Rollen waren dabei schnell verteilt zwischen dem potentiellen Front-Runner Mitt Romney und dem Rest des Bewerberfeldes, das fast ausschließlich aus Kandidaten bestand, die sich selbst rechts von Romney verorteten. Eine Ausnahme stellte wohl nur Jon Huntsman dar, der ehemalige Gouverneur von Utah und spätere amerikanische Botschafter in China. Huntsman stand mit manchen seiner Positionen tatsächlich links von seinen Mitbewerbern.

17 Benannt nach dem liberalen Republikaner Nelson D. Rockefeller, Gouverneur des Staates New York (1959 bis 1973) und Vize-Präsident der USA (1974 bis 1977).

18 Vgl. Arthur Paulson, Realignment and Party Revival. Understanding American Electoral Politics at the Turn of the Twenty-First Century, Westport 2000.

19 Vgl. Steven F. Hayward, The Age of Reagan. The Fall of the Old Liberal Order 1964-1980, New York 2001. 
Der eloquente und weltgewandte Ex-Diplomat hatte als Gouverneur gleichgeschlechtliche Lebenspartnerschaften unterstützt, stand auf dem Boden der Evolutionstheorie und leugnete nicht den Klimawandel. Im Gegensatz zu seinen Parteifreunden im US-Kongress hatte er für manche Maßnahmen der Obama-Regierung sogar freundliche Worte gefunden und dabei selbst die keynesianisch inspirierten Konjunkturprogramme des frisch gewählten Präsidenten gelobt. ${ }^{20}$ All das und seine generell konziliante Art gegenüber dem politischen Gegner brachten ihm zwar eine wohlwollende Berichterstattung in manch liberalem Leitmedium ein, machte ihn jedoch für die Republikanische Parteibasis und die meisten konservativen Parteispender höchst verdächtig; auf die allerdings sollte es ankommen bei dieser Vorwahl. Insofern war Huntsman einerseits ein von vornherein aussichtsloser Kandidat, der nach den ersten Vorwahlen schnell das Handtuch warf. Andererseits warf seine völlige Chancenlosigkeit im Bewerberfeld bereits ein bezeichnendes Licht auf die Veränderungen an der Republikanischen Parteibasis, denn Huntsman war insgesamt gewiss alles andere als ein Liberaler; vielmehr hatte er es als strikter Abtreibungsgegner und überzeugter Fiskalkonservativer geschafft, Gouverneur eines der konservativsten Bundesstaaten der USA zu werden. In seiner Amtszeit war er geradezu zum Darling der radikal-libertären Thinks Tanks im ganzen Land avanciert, wie etwa dem Cato Institute, da er rigoros Steuern gekürzt und Ausgaben gesenkt hatte. Aber Huntsman fehlte schon rein habituell etwas, nach dem viele Basisaktivisten geradezu zu lechzen schienen: eine aggressive populistische Ansprache, die die Kontraste zwischen den politischen Lagern markant und ohne unnötige Differenzierungen deutlich machte.

Die meisten seiner Mitbewerber verfügten über all das im Überfluss. Es war eine ziemlich bunte - und nach Meinung mancher Beobachter auch reichlich skurrile - Ansammlung Republikanischer Politiker, die sich seit Mitte des Jahres 2011 anschickten, die Nominierung ihrer Partei zu erhalten. Allerdings: wirklich bunt war das Kandidatenfeld nur aufgrund der erklecklichen Zahl von extravaganten Außenseitern, Polit-Abenteurern und flamboyanten Selbstdarstellern. In anderer Hinsicht spiegelte es eher die Einförmigkeit einer Partei wider, deren demographische Basis sich in den letzten Jahren bedenklich verengt hatte: weiß und männlich. Nur auf zwei Kandidaten aus dem Bewerberfeld traf das nicht zu, obgleich beide ideologisch durchaus nicht zur Mitte der Gesellschaft tendierten: Michele Bachmann und Herman Cain.

Bachmann, Kongressabgeordnete aus Minnesota, war in den Jahren nach 2009 schnell zur Ikone der Tea Party-Bewegung aufgestiegen, obgleich sie eigentlich eher im Lager der Christlichen Rechten zu verorten war. ${ }^{21}$ In den schnell aufeinander folgenden Favoritenernennungen der ersten Wahlkampfphase galt auch sie kurzeitig als besonders aussichtsreiche Kandidatin. Im Sommer 2011, in der Zeit der so genannten Invisible Primary, führte sie das Feld der Konkurrenten gemeinsam mit Romney in den Umfragen sogar kurzzeitig an. ${ }^{22} \mathrm{Als}$

20 Vgl. „Jon Huntsman: Picture Perfect”, in: The Economist vom 26. Mai 2011, http://www.economist.com/node/18744295 (Abruf am 15. Februar 2013).

21 Vgl. die ausführliche Reportage über Michele Bachmann von Ryan Lizza, Leap of Faith. The Making of a Republican Front-Runner, in: The New Yorker vom 15. August 2011, http://www.newyorker.com/reporting/2011/08/15/110815fa_fact_lizza?currentPage=all (Abruf am 15. Februar 2013).

22 Vgl. Public Policy Polling, Bachmann takes the lead, Pressemitteilung vom 19. Juli 2011, http:// www.publicpolicypolling.com/pdf/PPP_Release_National_0719.pdf (Abruf am 18. Februar 2013). 
es aber ernst wurde in der Invisible Primary und Bachmann sowie andere insbesondere in den Fernsehdebatten auf Herz und Nieren geprüft wurden, ging ihr Stern ziemlich schnell unter. Die Anführerin des Tea Party Caucus im Repräsentantenhaus produzierte eine Reihe bis heute legendärer verbaler Fehltritte, etwa als sie John Quincy Adams in die Reihe der amerikanischen Gründerväter stellte, obgleich dieser zum Zeitpunkt der Unabhängigkeitserklärung 1776 erst im zarten Alter von acht Jahren war; oder als sie den Geburtsort des großen Heroen der amerikanischen Wildwest-Kultur, John Wayne, mit dem eines Serienkillers gleichen Namens verwechselte. Mit Bachmann passierte, was mit anderen Favoriten des „Anyone but Romney“-Lagers später ebenfalls passieren sollte: So schnell sie nach oben gespült wurden, so rasch und unsentimental wurden sie von ihren enttäuschten Anhängern wieder fallen gelassen. Bei der ersten Vorwahl in Iowa - immerhin der Staat, in dem sie geboren wurde - erreichte Bachmann nur einen enttäuschenden 6. Platz. Kurz danach gab sie das Ende ihrer Kampagne bekannt.

Der andere medial viel beachtete Kandidat, für den das Prädikat „weiß und männlich“ nicht galt, schaffte es hingegen nicht einmal bis zur ersten Vorwahl: der afro-amerikanische Self-Made Millionär Herman Cain, der zwar bar jeder politischen Erfahrung war, dafür aber mit einem erstaunlichen Talent zur Selbstvermarktung ausgestattet. Auch Cain erlebte einen kurzen Frühling als Liebling der Tea Party, um dann umso heftiger abzustürzen. Das lag zum einen an behaupteten sexuellen Verfehlungen, aber auch daran, dass er den Anstrengungen des Wahlkampfes offenkundig nicht ganz gewachsen schien: So jedenfalls erklärte sein Wahlkampfmanager die sich häufenden Aussetzer Cains bei Interviews, wo er etwa in der Frage des Libyen-Konfliktes weder den Standpunkt des Präsidenten noch seinen eigenen verständlich erklären konnte. ${ }^{23}$ Cain überlebte politisch nicht einmal die Phase der Invisible Primary und zog seine Bewerbung noch vor den ersten Vorwahlen zurück.

Ein weiterer, eindeutig im konservativen Milieu der Partei verankerter Kandidat war der amtierende Gouverneur von Texas, Rick Perry. Auch er hatte es insbesondere in den Jahren nach 2008 zu einiger Prominenz gebracht, weil er in ungewöhnlich scharfer Form gegen die neue Regierung stritt, bisweilen gar - gewiss nicht ganz ernsthaft gemeint - mit der Sezession Texas' von den USA liebäugelte. ${ }^{24}$ Doch immerhin: anders als viele andere seiner extrem konservativen Mitbewerber hatte Perry als Gouverneur nicht nur bereits Erfahrungen in der Exekutive gesammelt, sondern dabei auch, zumindest auf dem Papier, eine erfolgreiche Bilanz vorzuweisen, da Texas in den Jahren der Wirtschaftskrise nach 2008 noch vergleichsweise gut da stand. Bald schon hatte sich indes auch Perry selbst ins Abseits manövriert.

23 Vgl. Richard A. Oppel, Cain Stumbles in Assessing Foreign Policy, in: New York Times vom 14. November 2012, http://www.nytimes.com/2011/11/15/us/politics/herman-cain-libya-commentsdraw-criticism.html?_r=0 (Abruf am 15. Februar 2013).

24 Perrys kontroverse Worte fielen bei einer Tea Party Rallye im Jahr 2009, als er sagte: „We've got a great union. There's absolutely no reason to dissolve it. But if Washington continues to thumb their nose at the American people, you know, who knows what might come out of that (...) When we came into the nation in 1845, we were a republic, we were a stand-alone nation. And one of the deals was, we can leave anytime we want. So we're kind of thinking about that again." Nach Obamas Wahlsieg 2012, als in einigen Bundesstaaten dann tatsächlich Petitionen unterschrieben wurden, die eine Loslösung von der Union forderten, sprach Perry sich allerdings sehr energisch dagegen aus. Vgl. Elyse Siegel, Rick Perry Takes Stand On Texas Secession Issue, in: Huffington Post vom 12. November 2012, http://www.huffingtonpost.com/2012/11/12/rick-perrysecession_n_2120453.html (Abruf am 10. Februar 2013). 
Seine Auftritte bei den Fernsehdebatten waren katastrophal, er wirkte nur mäßig vorbereitet und an den Details der Politik kaum interessiert. Schließlich produzierte er den vielleicht erinnerungswürdigsten Moment des gesamten Vorwahlkampfes, als er zunächst energisch die Abschaffung von drei überflüssigen Ministerien ankündigte, jedoch auf Nachfrage nur zwei davon benennen konnte.

Chancenlos, aber formell wesentlich länger im Rennen, war Ron Paul, der bereits zum dritten Mal seinen Hut in den Ring geworfen hatte. ${ }^{25}$ Wäre die Tea Party tatsächlich aus ihren offiziellen Entstehungsgründen begreifbar - dem Protest gegen den Bailout der Banken und Autoindustrie, den Konjukturprogrammen - dann hätte Paul eigentlich ihr großer Held sein müssen. Kaum jemand anders hatte so vehement, so ausdauernd und auch ideologisch konsistent gegen „Big Government“ gekämpft wie der Kongressabgeordnete aus Texas. ${ }^{26}$ Und Paul besaß tatsächlich eine überzeugte Schar von jungen, hochmotivierten Graswurzelaktivisten. Doch mit anderen Positionen stand er auch bei dieser Kampagne weit entfernt vom Mainstream der Partei, denn er war eben zeitlebens ein wirklich überzeugter Libertärer gewesen und konnte daher mit vielen Positionen, die innerhalb der Republikanischen Partei mehrheitsfähig sind, wenig anfangen. Paul vertrat eine isolationistische Außenpolitik, befürwortete die Freigabe von weichen Drogen wie Marihuana und abgesehen von seiner Opposition gegen die Abtreibung nahm er auch bei den „social issues“ fast ausschließlich liberale Positionen ein. Programmatisch war all das konzise und in sich logisch, aber es machte ihn in weiten Teilen der Partei zum Außenseiter - auch bei der Tea Party. Diese hatte nämlich seit ihrer Entstehungsphase 2009 einen interessanten Weg eingeschlagen: Als heterogene Bewegung ohne eindeutiges programmatisches, geschweige denn organisatorisches Zentrum war sie zum Sammelbecken aller möglichen konservativen Gruppen und ihrer Anliegen geworden, die zwar der Protest gegen Obama einte, ansonsten aber ganz unterschiedliche Aktivistenbiographien verband. ${ }^{27}$ Mittlerweile bezeichneten sich auch Menschen als Tea Party-Unterstützer, die eher aus dem religiös-konservativen Umfeld stammten. Bei ihnen konnte Paul mit seinen Positionen kaum punkten und eignete sich folglich auch nicht zum Anti-Romney.

In der besonders raschen Abfolge von Aufstieg und Fall konservativer Hoffnungsträger manifestierte sich die nervöse Unsicherheit eines großen Teiles der Parteibasis, der zwar in irgendeiner Weise das diffuse Gefühl hatte, dass Romney nicht wirklich aus der Mitte ihrer Bewegung käme, aber keine überzeugende Alternative zu ihm finden konnte. Tatsächlich erhielten Romneys Konkurrenten auch kaum die Gelegenheit, in programmatischer Hinsicht ein tragfähiges konservatives Gegenangebot zu ihm aufzubauen - denn er hatte längst alle moderaten Positionen geräumt. Und zwar schon seit geraumer Zeit: Erinnert sei daran, dass Romney noch 2008 als konservative Alternative zu John McCain gehandelt wurde. Seitdem war er aber keineswegs nach links beziehungsweise in die Mitte gerückt, sondern hatte auf einzelnen Politikfeldern sein konservatives Profil weiter geschärft. 2012 distanzierte er sich endgültig von der Politik, die er als Gouverneur des Bundesstaates Massachusetts betrieben hatte, vor allem von seiner damaligen Gesundheitspolitik, die in vielerlei Hinsicht ein Vor-

25 Allerdings war Ron Paul 1988 als Kandidat der Libertarian Party angetreten.

26 Vgl. Wendy Kaminer, If Voters Cared About Liberty, Ron Paul Would Be the Frontrunner, in: The Atlantic vom 4. Januar 2012, http://www.theatlantic.com/politics/archive/2012/01/if-voterscared-about-liberty-ron-paul-would-be-the-frontrunner/250880/ (Abruf am 16. Februar 2013).

27 Vgl. dazu unter anderem Ronald P. Formisano, The Tea Party: A Brief History, Baltimore 2012. 
bild für Obamas Reformen gewesen war. Später, im Hauptwahlkampf, sollte sich diese Wende noch einmal rächen, aber in den Primaries gelang es ihm damit tatsächlich, die Vorbehalte gegen ihn ein Stück weit zu neutralisieren, indem er der Tea Party und anderen konservativen Gruppen weit entgegenkam. Damit setzte Romney fort, was auch andere etablierte Parteieliten vor ihm getan hatten: eine Art vorauseilende Appeasement-Politik gegenüber einer immer schwerer zu kontrollierenden Parteibasis. So zeigte sich in der Vorwahl der Republikaner 2012 auch die Homogenisierung der Partei insgesamt, in der die wirklichen ideologischen Unterschiede - sieht man von Ron Paul und vielleicht Jon Huntsman ab - nur noch gering waren. In den 1960er und 1970er Jahren war es wirklich noch ein Konflikt zwischen konservativen und moderaten Kräften gewesen; aber dieser Kampf war längst entschieden, da letztere in der Folgezeit fast komplett aus der Partei verschwanden. Jetzt entzündete sich der Konflikt eher zwischen jenen, die noch in den Dimensionen von Gesetzesvorhaben oder realer Politik dachten und jenen, die ein Ventil für ihren Protest suchten, eine Art Anti-Politik anstrebten und für die Kompromiss ein Schimpfwort geworden war - der Krieg zwischen den „Professionals“ und den „Protesters“, wie es der konservative New York Times Kolumnist David Brooks treffend beschrieb. ${ }^{28}$ Gerade diese eigentlich ideologische Homogenität aber führte innerhalb des Bewerberfeldes zu immer neuen, noch populistischeren Attacken gegen Mitt Romney, um eine Differenz herauszustellen, die in Wahrheit kaum noch vorhanden war.

Am ehesten beherrschten dieses Spiel wohl jene zwei Kandidaten, die wenigstens zeitweise den Eindruck erweckten, als könnten sie dem „inevitable candidate“ Romney die Nominierung vielleicht doch streitig machen: Newt Gingrich und Rick Santorum. Nicht zufällig waren beide - trotz ihres Hanges zur politischen Provokation und obgleich sie durchaus begabte Populisten waren - eben gerade keine plötzlich auftauchenden politischen ShootingStars oder Provinz-Fürsten, sondern erfahrene Washingtoner Schwergewichte, im Grunde Teil jenes Polit-Establishments, das ihre Anhänger zutiefst verachteten.

Newt Gingrich, der fast schon legendär-berüchtigte Anführer der „Republikanischen Revolution“ des Jahres 1994, als die Kongresswahlen eine ganze Reihe sehr konservativer Abgeordnete in den Republikanischen Caucus spülte ${ }^{29}$, war der Partei-Intellektuelle im Feld. Der ehemalige Geschichtsprofessor zeichnete gern die großen Linien konservativer Ideologie und trat jede Woche mit einer neuen konkreten Politik-Idee auf den Plan, auch wenn nicht alles immer ganz ausgereift schien. Aber Gingrich hatte nicht nur eine Reihe von unerfreulichen Skandalen im Gepäck, sondern auch sein Temperament nicht immer im Griff und neigte dann, einmal in Fahrt gekommen, zu schwer haltbaren Übertreibungen. Zudem war seine politische Bilanz insgesamt durchaus gemischt, denn er hatte in den Clinton-Jahren - nachdem der erste Furor über die konservative Revolution verflogen war - Kompromisse geschlossen und Positionen bezogen, die im Jahre 2012 im Zuge der doktrinären Verhärtung der Partei als moderat oder schon liberal galten. Überdies war er nach seinem Ausscheiden aus dem Kongress ein sehr gut bezahlter Lobbyist gewesen, unter anderem für die Hypothekenbank Freddie Mac - einem jener Institute, die nach der Immobilienkrise 2008 mit den

28 Vgl. David Brooks, The Possum Republicans, in: New York Times vom 27. Februar 2012, http:// www.nytimes.com/2012/02/28/opinion/brooks-the-possum-republicans.html (Abruf am 19. Februar 2013).

29 Vgl. John Micklethwait / Adrian Wooldridge, The Right Nation. Conservative Power in America, New York 2005, S. $94-131$. 
Geldern des amerikanischen Steuerzahlers gerettet werden mussten. Aber Gingrich - und das sicherte ihm in diesem Wahlkampf eine überzeugte Kernanhängerschaft - verstand auf der Klaviatur des konservativen Populismus wie kein Zweiter zu spielen, und seine heftigen Invektiven gegen die Regierung, die „liberalen Medien“ und schließlich Mitt Romney machten ihn 2012 in Teilen der Basis wieder zu einer respektablen Figur. ${ }^{30}$ Er fühlte sich ständig benachteiligt, klein gehalten, stets unter Wert gehandelt und hatte so Ressentiments entwickelt, die viele seiner Anhänger ebenfalls empfanden.

Rick Santorum, der frühere Senator von Pennsylvania, war im Grunde ein eindeutiger Vertreter des sozial-konservativen Flügels der Partei und hatte sich bereits in der Vergangenheit als erbitterter Abtreibungsgegner und Homosexuellen-Feind profiliert. Mittlerweile stand er zwar - wie alle, die in der Republikanischen Partei etwas werden wollten - auch in der Wirtschafts- und Sozialpolitik den libertären, orthodoxen Standpunkten der Partei näher, stritt für Steuersenkungen und gegen die Bevormundung durch die Zentralregierung in Washington; im Gegensatz zu anderen hatte er sich aber nie von George W. Bush's Konzept des „compassionate conservatism“ distanziert. ${ }^{31}$ Wenn Gingrich gern Geschichtsstunden gab, dann inszenierte Santorum sich mit Vorliebe als Vertreter des „average man“ und punktete vor allem mit einem scharfen Anti-Intellektualismus, der vor ihm schon Sarah Palin nach oben gespült hatte ${ }^{32}$; dass Obama meinte, jeder Amerikaner solle eine College-Ausbildung anstreben, empfand er als elitäre Bevormundung und Anmaßung: „What a snob!“

Der Konflikt zwischen dem Front-Runner Mitt Romney und Newt Gingrich sowie Rick Santorum als seinen konservativen Gegenspielern sollte die Dramaturgie des folgenden Wahlkampfes prägen.

\section{Der Verlauf des Wahlkampfs: Mitt Romneys beständiger Weg zur Nominierung}

\subsection{Die frühen Entscheidungen}

Den ersten Entscheidungen, insbesondere denen in Iowa und New Hampshire, kommt traditionell eine hervorgehobene Stellung zu, obwohl hier nur über einen Bruchteil der Delegiertenstimmen auf dem Parteitag entschieden wird - 2012 wurden weniger als zwei Prozent der Parteitagsdelegierten aus diesen beiden Staaten entsandt. ${ }^{33}$ Dennoch richten besonders national weniger bekannte Kandidaten oder solche, die nicht von Anfang an über die finanziellen Mittel oder die Parteiunterstützung verfügen, um eine landesweite Kampagne auf die Beine zu stellen, ihre Bemühungen oft auf diese frühen Vorwahlstaaten. 2012 verfolgte Rick Santorum diese Strategie. Mit weniger Ressourcen ausgestattet, setzte er auf

30 Vgl. Richard McGregor, The Rise of Gingrich and the "Elite Media", in: Financial Times vom 28. November 2011, http://www.ft.com/cms/s/0/bd57cfaa-19e2-11e1-b9d7-00144feabdc0. html\#axzz2LKqNqm3y (Abruf am 17. Februar 2013).

31 Vgl. Michael Gerson, The Return of Compassionate Conservatism, in: Washington Post vom 5. Januar 2012, http://articles.washingtonpost.com/2012-01-05/opinions/35441925_1_libertarianism-rick-santorum-conservatism (Abruf am 18. Februar 2013).

32 Vgl. Torben Lütjen, Die populistische Versuchung. Zur Aktualität der Tradition des Anti-Intellektualismus in den USA, in: Universitas, 65. Jg. (2010), H. 7, S. $664-675$.

33 Vgl. The Green Papers, 2012 Chronological Cumulative Allocation of Delegates, http://www. thegreenpapers.com/P12/ccad.phtml (Abruf am 13. Februar 2013). 
einen Überraschungssieg in Iowa, um „Momentum“ zu generieren, das ihm mehr positive Berichterstattung, mehr Spenden und eine größere Legitimität für seine Kandidatur bescheren sollte. Völlig mittellos stand indes auch Santorums Kampagne nicht da, zumal er von dem Super PAC „Red, White, and Blue Fund“ unterstützt wurde, das konservative Wähler dazu aufrief, sich hinter ihm als dem einzig wahren konservativen Kandidaten zu vereinigen. ${ }^{34}$ Als zusätzliches Plus für Santorum wirkte, dass sich in Iowa die Mehrheit der CaucusTeilnehmer als evangelikale Christen bezeichnete. ${ }^{35}$ Tatsächlich gewann Santorum die Iowa Caucuses nach einer Neuauszählung der Stimmen knapp gegen Romney, dessen zweiter Platz als Achtungserfolg interpretiert wurde. Michele Bachmann beendete, wie bereits gesagt, infolge ihres schwachen Wahlergebnisses ihre Kandidatur. Sie musste einsehen, dass Santorum dieselben Strömungen in der Partei ansprach wie sie und damit ungleich erfolgreicher war. ${ }^{36}$ Eigentlich also gute Nachrichten für ihn, insbesondere im Hinblick auf die bevorstehende Vorwahl in South Carolina, wo Social Conservatives und Evangelikale einen großen Teil der Republikanischen Basis ausmachen. ${ }^{37}$ Doch zuvor musste Santorum sich den liberaleren Wählern in New Hampshire stellen, und dies bereits am 10. Januar, eine Woche nach den Iowa Caucuses - wenig Zeit also um Fahrt aufzunehmen. Erschwerend kam die Tatsache hinzu, dass New Hampshire als „Romney Territory“ gelten konnte, da dieser den Nachbarstaat Massachusetts von 2003 bis 2007 als Gouverneur regiert hatte. Und so war es Romney, der diese traditionell erste Primary mit 39,3 Prozent der Stimmen für sich entschied, gefolgt von Ron Paul und Jon Huntsman. Letzterer hatte alle Energien, Ressourcen und Hoffnungen auf seinen Durchbruch dort gerichtet, weshalb er sich am 16. Januar folgerichtig aus dem Rennen zurückzog und eine Wahlempfehlung für Romney abgab. ${ }^{38}$

Die ersten Ergebnisse in diesen Staaten ließen zumindest vermuten, dass eine konservative Alternative zu Romney denkbar gewesen wäre - wenn ein Kandidat die Vorbehalte gegen ihn hätte bündeln können. In South Carolina am 21. Januar siegte jedoch nicht Rick Santorum, der als Sieger von Iowa inzwischen zunehmend kritische, weil tiefergehende Berichterstattung der Medien über sich ergehen lassen musste. Mit dem deutlichen Ergebnis von 40,4 Prozent der Stimmen gewann stattdessen der neue Aufsteiger Newt Gingrich. Dieser hatte in der Zwischenzeit das Endorsement des ausgeschiedenen Rick Perry erhalten. ${ }^{39}$ Vor allem hatte er jedoch seine Medienpräsenz durch gute Auftritte bei zwei Fernsehdebatten der Republikanischen Kandidaten im Vorfeld der South Carolina Primary steigern können. Außerdem attackierte sein Super PAC Romneys frühere Tätigkeit für die Investmentfir-

34 Vgl. Shushannah Walshe, Rick Santorum Super PAC Launches New Iowa Ad, http://abcnews. go.com/blogs/politics/2011/12/rick-santorum-super-pac-launches-new-iowa-ad (Abruf am 11. Februar 2013).

35 Vgl. John Sides / Lynn Vaureck, All In, in: dies. (Hrsg.), The Gamble: Choice and Chance in the 2012 Presidential Election, Princeton 2012, Online-Vorabdruck, http://press.princeton.edu/chapters/s4-10052.pdf (Abruf am 24. Januar 2013), S. 3 - 59, S. 12.

36 Vgl. Emily Schultheis, Michele Bachmann Drops 2012 Campaign for President, in: Politico vom 4. Januar 2012, http://www.politico.com/news/stories/0112/71078.html (Abruf am 13. Februar 2013).

37 Vgl. ebenda.

38 Vgl. CBSNews, Huntsman Suspends Campaign, Endorses Romney, http://www.cbsnews. com/8301-250_162-57359519/huntsman-suspends-campaign-endorses-romney/ (Abruf am 13. Februar 2013).

39 Vgl. Peter Hamby, Perry Drops Out, Endorses Gingrich, http://edition.cnn.com/2012/01/19/ politics/perry-dropping-out (Abruf am 13. Februar 2013). 
ma Bain Capital, was den Front-Runner unter Rechtfertigungsdruck setzte. ${ }^{40}$ Er wurde abgeschlagener Zweiter (mit 27,8 Prozent) und Gingrich, dessen Kampagne nach seinem schwachen vierten Platz in New Hampshire schon als fast aussichtslos erschien, etablierte sich nun als erster Rivale Romneys und Vertreter der konservativen Strömungen in der Partei. ${ }^{41}$ Viele Delegierte nahm Gingrich allerdings nicht mit. Aufgrund der Bestrafung South Carolinas durch das RNC erhielt er lediglich 20 Delegiertenstimmen für seinen Sieg, Romney bekam noch fünf. ${ }^{42}$

Dessen Auftritt in einer weiteren TV-Debatte nur zwei Tage später half, seinen eigenen Abwärtstrend zu stoppen. So konnte Romney mit Zuversicht auf die nächste große Entscheidung in Florida schauen, wo er dank seiner überlegenen Organisation, dem überaus umtriebigen Super PAC „Restore Our Future“ und der für ihn wieder günstigeren Zusammensetzung der potenziellen Wählerschaft auch gewinnen sollte. ${ }^{43}$ Gingrich wartete zudem vergeblich darauf, dass sich führende Republikaner öffentlich zu ihm als Kandidaten bekannten. Vielmehr wurde die Aussicht auf ihn als möglichen Präsidentschaftskandidaten vielerorts im Parteiestablishment mit Sorge betrachtet; Gingrich hatte sich mit seiner unberechenbaren und unkontrollierten Art in seinen drei Jahrzehnten in Washington offenbar ein paar Feinde zu viel gemacht. ${ }^{44}$

\subsection{Die Zwischenphase}

Auf Mitt Romneys Sieg in den Nevada Caucuses folgte die Woche Rick Santorums, der mit Colorado, Minnesota und Missouri in drei weiteren Bundesstaaten gewann, deren Republikanische Wählerschaft eine für ihn günstige Zusammensetzung aufwies (vergleichsweise viele Evangelikale und „strong conservatives“). Allerdings handelte es sich bei den ersten beiden um "non-binding caucuses“, in denen die Delegierten nicht durch das Ergebnis bereits auf den Wahlsieger verpflichtet sind. Die Vorwahl in Missouri hatte gar keinen Einfluss auf die Verteilung der Delegiertenstimmen. Über diese wurde in den Wochen darauf nach dem Caucus-Convention-System entschieden. Santorum konnte sich der Delegierten also im Moment des Wahlsieges nicht sicher sein. ${ }^{45}$

Die nächsten Primaries am 28. Februar in Michigan und Arizona waren hinsichtlich der Zusammensetzung der Wählerschaft wieder Romney-freundlicher, so dass er beide Vorwahlen gewinnen konnte. Im Nachhinein ist sein Sieg in Michigan als Klimax der Kampagne bezeichnet worden. In jedem Fall nahm er Santorum - unterstützt von einer kritischeren Medienöffentlichkeit - erneut das schwache Momentum, das dieser nach seinen Erfolgen

40 Vgl. John Sides / Lynn Vavreck, a.a.O. (Fn. 35), S. 17.

41 The New York Times, South Carolina Republican Primary, http://elections.nytimes.com/2012/ primaries/states/south-carolina, (Abruf am 12. Februar 2013).

42 Ebenda.

43 Vgl. John Sides / Lynn Vavreck, a.a.O. (Fn. 35), S. 26.

44 Vgl. Richard W. Stevenson, Gingrich Insurgency Tests Party's Old Guard, in: The New York Times (The Caucus - The Politics and Government Blog) vom 26. Januar 2012, http://thecaucus.blogs. nytimes.com/2012/01/26/gingrich-insurgency-tests-partys-old-guard/ (Abruf am 17. Februar 2013); John Sides / Lynn Vavreck, a.a.O. (Fn. 35), S. 29.

45 Vgl. John Sides / Lynn Vavreck, a.a.O. (Fn. 35), S. 34. 
verspürt haben mochte. ${ }^{46}$ Dieser Sieg scheint Mitt Romney tatsächlich von dem sehr wahrscheinlichen Gewinner zu einem fast sicheren gemacht zu haben, wie Nate Silver seinerzeit in seinem Blog FiveThirtyEight schrieb. ${ }^{47}$ In der Folge gab Romney seine Führung im Gallup Tracking Poll nicht mehr ab, und die Berichterstattung über den Vorwahlkampf schaffte es immer seltener an die Spitze der Nachrichtenagenda - ein Indiz für die nachlassende Spannung des Rennens. ${ }^{48}$ Den Verlauf der restlichen Vorwahlsaison charakterisiert Silver als „war of attrition“ - ein Zermürbungskrieg, den Romney aufgrund seiner kontinuierlichen Zugewinne an Delegiertenstimmen, der Unterstützung durch das Establishment, seines erfolgreicheren Fundraisings und seines erfahrenen Wahlkampfteams auch gewinnen würde. ${ }^{49}$ Hätte einer von Romneys verbliebenen Konkurrenten über mehrere Wochen hinweg einige Vorwahlsiege hintereinander einfahren können, wäre er vielleicht tatsächlich als chancenreicher Kandidat erschienen und hätte durch die intensivere Medienberichterstattung auch bei potenziellen Spendern populärer werden können. Doch für diese Art des Momentums bot schon der Wahlkalender keine günstigen Voraussetzungen. Auf Staaten, in denen die Zusammensetzung der Republikanischen Wählerschaft Gingrich oder Santorum begünstigte, folgten stets bald solche, in denen ein Erfolg Romneys wahrscheinlicher war. ${ }^{50}$

Weil zudem der Klimax - im Nachhinein betrachtet - bereits überschritten war, nahm auch der so genannte Super Tuesday am 6. März keine so herausragende Stellung mehr ein. Zwar fanden zeitgleich sieben Primaries (Georgia, Massachusetts, Ohio, Oklahoma, Tennessee, Vermont und Virginia) und drei Caucuses (Alaska, Idaho und North Dakota) statt. Doch war dies wesentlich weniger als 2008. ${ }^{51}$ Newt Gingrich gewann an diesem Tag lediglich seinen Heimatstaat Georgia, Rick Santorum war in drei Staaten erfolgreich, Mitt Romneymit der Unterstützung seines Super PACs, das in Sachen Wahlwerbung die Unterstützer Santorums und Gingrichs in den Schatten stellte - in den restlichen sechs. ${ }^{52}$ Da der Super Tuesday aber auch keinen überlegenen Triumph für Romney brachte, setzte sich der Abnutzungskampf fort. ${ }^{53}$

Bis Ende März konnte Santorum zwar noch in vier Staaten gewinnen und Romney, der fünfmal siegte, zumindest bei der Anzahl der gewonnenen Staaten im Monat März die Stirn bieten. Doch die Unterstützung seitens des Establishments vermochte er dadurch ebenso wenig zu erringen, wie er Rückhalt außerhalb seiner Kernklientel genoss. Wirklich breite Teile des großen „Republican Tent“ konnte nur Romney ansprechen. Als Romney Ende des Monats auch noch die Endorsements von strammen Konservativen wie Marco Rubio und

46 Vgl. ebenda, S. 35.

47 Vgl. Nate Silver, Romney's Michigan Win Stands as Climactic Moment in Campaign, in: The New York Times (FiveThirtyEight - Nate Silver's Political Calculus) vom 4. April 2012, http://fivethirtyeight.blogs.nytimes.com/2012/04/04/romneys-michigan-win-stands-as-climactic-moment-incampaign/ (Abruf am 17. Februar 2013).

48 Vgl. ebenda.

49 Vgl. ebenda.

50 Vgl. John Sides / Lynn Vavreck, a.a.O. (Fn. 35), S. 38.

51 Am damaligen Super Tuesday, dem 5. Februar 2008 fanden in 20 Bundesstaaten Nominierungsentscheidungen der Republikaner statt.

52 Vgl. RealClearPolitics, 2012 Republican Popular Vote, http://www.realclearpolitics.com/ epolls/2012/president/republican_vote_count.html (Abruf am 17. Februar 2013).

53 Vgl. Jeff Zeleny, With No Knockout Punch, a Bruising Battle Plods On, in: The New York Times vom 7. März 2012, https://www.nytimes.com/2012/03/07/us/politics/no-super-tuesday-knockout-punch.html (Abruf am 17. Februar 2013). 
Tabelle 2: Ergebnisse der Republikanischen Primaries und Caucuses 2012 (in Prozent)

\begin{tabular}{|c|c|c|c|c|c|}
\hline \multirow{2}{*}{ Primaries / Caucuses } & \multirow{2}{*}{ Datum } & \multicolumn{4}{|c|}{ Stimmenanteile für } \\
\hline & & Romney & Santorum & Gingrich & Paul \\
\hline Iowa (C) & 3. Januar & 25 & 25 & 13 & 21 \\
\hline New Hampshire (P) & 10. Januar & 39 & 9 & 9 & 23 \\
\hline South Carolina (P) & 21. Januar & 28 & 17 & 40 & 13 \\
\hline Florida (P) & 31. Januar & 46 & 13 & 32 & 7 \\
\hline Nevada (C) & 4. Februar & 50 & 10 & 21 & 19 \\
\hline Colorado (C) & 7. Februar & 35 & 40 & 13 & 12 \\
\hline Minnesota (C) & 7. Februar & 17 & 45 & 11 & 27 \\
\hline Missouri (P) & 7. Februar & 25 & 55 & - & 12 \\
\hline Maine (C) & 11. Februar & 38 & 18 & 7 & 36 \\
\hline Arizona $(\mathrm{P})$ & 28. Februar & 47 & 27 & 16 & 9 \\
\hline Michigan (P) & 28. Februar & 41 & 38 & 7 & 12 \\
\hline Wyoming (C) & 29. Februar & 39 & 32 & 8 & 21 \\
\hline Washington (C) & 3. März & 38 & 24 & 10 & 25 \\
\hline Alaska (C) & 6. März & 32 & 30 & 13 & 24 \\
\hline Georgia (P) & 6. März & 26 & 20 & 47 & 7 \\
\hline Idaho (C) & 6. März & 62 & 18 & 2 & 18 \\
\hline Massachusetts (P) & 6. März & 72 & 12 & 5 & 10 \\
\hline North Dakota (C) & 6. März & 24 & 40 & 9 & 28 \\
\hline Ohio (P) & 6. März & 38 & 37 & 15 & 9 \\
\hline Oklahoma (P) & 6. März & 28 & 34 & 28 & 10 \\
\hline Tennessee $(\mathrm{P})$ & 6. März & 28 & 37 & 24 & 9 \\
\hline Vermont (P) & 6. März & 40 & 24 & 8 & 25 \\
\hline Virginia (P) & 6. März & 60 & - & - & 41 \\
\hline Kansas (C) & 10. März & 21 & 51 & 14 & 13 \\
\hline Alabama (P) & 13. März & 29 & 35 & 29 & 5 \\
\hline Hawaii (C) & 13. März & 45 & 25 & 11 & 19 \\
\hline Mississippi (P) & 13. März & 31 & 33 & 31 & 4 \\
\hline Illinois $(\mathrm{P})$ & 20. März & 47 & 35 & 8 & 9 \\
\hline Louisiana (P) & 24. März & 27 & 49 & 16 & 6 \\
\hline Dist. of Columbia (P) & 3. April & 70 & - & 11 & 12 \\
\hline Maryland (P) & 3. April & 49 & 29 & 11 & 10 \\
\hline Wisconsin $(\mathrm{P})$ & 3. April & 44 & 37 & 6 & 11 \\
\hline Connecticut (P) & 24. April & 67 & 7 & 10 & 14 \\
\hline Delaware (P) & 24. April & 57 & 6 & 27 & 11 \\
\hline New York (P) & 24. April & 63 & 10 & 13 & 15 \\
\hline Pennsylvania (P) & 24. April & 58 & 18 & 10 & 13 \\
\hline Rhode Island (P) & 24. April & 63 & 6 & 6 & 24 \\
\hline Indiana $(P)$ & 8. Mai & 65 & 13 & 7 & 16 \\
\hline North Carolina (P) & 8. Mai & 66 & 10 & 8 & 11 \\
\hline West Virginia (P) & 8. Mai & 70 & 12 & 6 & 11 \\
\hline Nebraska (P) & 15. Mai & 71 & 14 & 5 & 10 \\
\hline Oregon $(\mathrm{P})$ & 15. Mai & 71 & 9 & 5 & 13 \\
\hline Arkansas (P) & 22. Mai & 68 & 13 & 5 & 13 \\
\hline Kentucky (P) & 22. Mai & 67 & 9 & 6 & 13 \\
\hline Texas $(\mathrm{P})$ & 29. Mai & 69 & 8 & 5 & 12 \\
\hline California (P) & 5. Juni & 79 & 5 & 4 & 10 \\
\hline Montana (P) & 5. Juni & 68 & 9 & 4 & 14 \\
\hline New Jersey (P) & 5. Juni & 81 & 5 & 3 & 10 \\
\hline New Mexico (P) & 5. Juni & 73 & 11 & 6 & 10 \\
\hline South Dakota (P) & 5. Juni & 66 & 11 & 4 & 13 \\
\hline Utah $(\mathrm{P})$ & 26. Juni & 93 & 2 & 1 & 5 \\
\hline
\end{tabular}

Anmerkung: Der Fettdruck zeigt den Sieger der jeweiligen Primary beziehungsweise des jeweiligen Caucus. Aufgrund der starken Rundung kann die Summe der Werte in einer Zeile mehr als 100 Prozent betragen. Ein Strich (-) bedeutet, dass der Name des Kandidaten nicht auf dem Wahlzettel stand. Nicht aufgeführt sind die Außengebiete der Vereinigten Staaten (Puerto Rico, die Amerikanischen Jungferninseln, Guam, die Nördlichen Mariannen und Amerikanisch-Samoa).

Quelle: http://www.uselectionatlas.org (Abruf am 25. Februar 2013); Rhodes Cook, Romney as VoteGetter, in: The Rhodes Cook Letter, 13. Jg. (2012), H. 2, S. 3-11, S. 10. 
Paul Ryan erhielt und Santorum öffentlich zur Aufgabe ermutigt wurde, sprach nichts dafür, dass dieser seine Kampagne noch lange würde fortführen können. ${ }^{54}$

\subsection{Die finale Phase}

Am 10. April erklärte Santorum seine Kampagne für beendet. Er hatte insgesamt sechs Vorwahlen und fünf Caucuses gewonnen, Romney hatte bis zu diesem Zeitpunkt insgesamt 19 Vorwahlen und Caucuses für sich entscheiden können. Santorum war vor allem der Kandidat des ländlichen Amerika, wie eine Betrachtung der Stimmenverteilung auf CountyEbene deutlich macht, Romney war hingegen am erfolgreichsten in urbanen Gegenden. ${ }^{55}$

Von nun an gab es in den verbleibenden 19 Einzelstaaten nur noch einen Gewinner: Mitt Romney. Am 25. April stellte sich das RNC hinter ihn als designierten Herausforderer Präsident Obamas, woraus Newt Gingrich am 2. Mai schließlich die Konsequenzen zog und sich ebenfalls aus dem Rennen zurückzog. Er hatte lediglich die Vorwahlen in South Carolina und Georgia für sich entscheiden und insgesamt 143 Delegierte für die Convention sammeln können. ${ }^{56}$ Am 14. Mai gab Ron Paul bekannt, keine weiteren Ressourcen in die noch ausstehenden Vorwahlen zu investieren, sondern stattdessen um die Unterstützung der noch ungebundenen Delegierten („unpledged delegates“) zu werben, was ihm in drei Bundesstaaten auch gelang. Bis Romney die 1.144 für eine Parteitagsmehrheit erforderlichen Delegiertenstimmen beisammen hatte, dauerte es jedoch bis zur Vorwahl im bevölkerungsreichen Texas am 29. Mai. Die darauf noch folgenden Nominierungsentscheidungen in sechs weiteren Staaten besaßen somit nur noch akklamatorischen Charakter.

Der Front-Runner hatte sich also durchgesetzt. Es gewann der Kandidat mit dem größten Wahlkampfbudget, dem finanzkräftigsten Super PAC und der professionellsten Wahlkampforganisation; neben dem erfolgreichen „Air War“ über die Massenmedien hatte Romney in vielen Staaten auch ein besseres „Ground Game“ als seine Konkurrenten.

Allerdings trugen die neuen Spielregeln in Kombination mit den Aktivitäten von Gruppen von außen (Super PACs) vermutlich dazu bei, dass es auch keine Blitzentscheidung wie 2008 gab. Dies lag eher an den Regeln bezüglich der Terminierung, weniger an den Veränderungen bei der Delegiertenverteilung. ${ }^{57}$ Dennoch hatten 2012 mehrere Kandidaten die Chance, sich den Wählern über mehrere Wochen zu präsentieren. Dabei konnten die Sanktionen des RNC das Frontloading nicht ganz verhindern, wie der erneut sehr frühe Start der Vorwahlsaison belegt.

Betrachtet man die Wählerschaft in den Vorwahlen der GOP, so hatte Romney von vornherein zumindest durchschnittliche Beliebtheitswerte und war für viele, wenn nicht die erste, so doch die beste zweite Wahl, mit der man sich auch anfreunden konnte. ${ }^{58}$ In jedem

54 Vgl. John Sides / Lynn Vavreck, a.a.O. (Fn. 35), S. 38.

55 Vgl. Rhodes Cook, Romney as Vote-Getter, in: The Rhodes Cook Letter, 13. Jg. (2012), H. 2, S. 3 -11 , S. $9 \mathrm{ff}$.

56 Vgl. The Green Papers, Election 2012. Presidential Primaries, Caucuses, and Conventions, http:// www.thegreenpapers.com/P12/ (Abruf am 17. Februar 2013).

57 Siehe dazu Josh Putnam / John Sides, Republican Rules Are Not to Blame for Primary War, http:// www.bloomberg.com/news/2012-03-22/republican-rules-are-not-to-blame-for-primary-war.html (Abruf 24. Januar 2013).

58 Vgl. John Sides / Lynn Vavreck, a.a.O. (Fn. 35), S. 40. 
Fall wurde er im Frühjahr von den Republikanischen Wählern als Kandidat mit den größten Chancen gegen Obama angesehen. ${ }^{59}$ Es gibt zudem Anhaltspunkte dafür, dass Romney als ideologisch gar nicht so weit entfernt von dem Punkt eingeordnet wurde, an dem sich diese in den Vorwahlen selbst platzierten. ${ }^{60}$ Wenn er auch nicht der Kandidat war, der ihnen ideologisch am nächsten stand, so erschien einer Mehrheit der Abstand dennoch nicht so groß, dass man ihn aus ideologischen Gründen hätte ablehnen müssen. ${ }^{61}$ Zudem entschieden sich viele Romney-Wähler anscheinend nicht ausschließlich auf Basis der Ideologie, sondern gewichteten die „electability“, also seine Chancen, in der Hauptwahl Obama abzulösen, stärker. ${ }^{62}$ Das mag auch eine Erklärung für sein teilweise überraschend gutes Abschneiden bei Tea Party-Anhängern sein. ${ }^{63}$ Laut den Exit Polls, die im Auftrag von CBS News in einer Reihe von Einzelstaaten durchgeführt wurden, stimmten die „Strong Tea Party Supporters“ zu 33 Prozent für Romney, gefolgt von Santorum mit 31, Gingrich mit 28 und Ron Paul mit sieben Prozent. ${ }^{64}$ Dennoch offenbarte Romney auch Schwächen in einigen Gruppen: Sowohl bei den weißen Evangelikalen als auch bei jenen, die sich als sehr konservativ bezeichneten, lag Santorum vorn. ${ }^{65}$

\section{Parteibasis und Establishment: Geeint im Ziel, Obama abzulösen}

Bedeutet die Nominierung Mitt Romneys also, dass das Establishment die Kontrolle über die Republikanische Partei zurückgewonnen hat? In jedem Fall war es ein Sieg, der die meisten Gesetzmäßigkeiten amerikanischer Vorwahlkämpfe zu bestätigen scheint, nicht nur weil Romneys Wahlkampfmaschine der Konkurrenz unendlich überlegen war. Durchgesetzt hatte sich am Ende eben auch der Kandidat, der die meiste Unterstützung vom Republikanischen Establishment in Washington und in den Einzelstaaten erhalten hatte. Insofern gilt noch immer: „The Party decides." 66

Dabei stimmte es, dass viele konservative Aktivisten bis zum Ende nicht recht warm werden wollten mit Romney, was einerseits erklärt, wie urplötzlich einzelne seiner Konkurrenten, die sich rechts von ihm positionierten, zu gefeierten Hoffnungsträgern avancieren konnten. Andererseits: In der Regel ging es dabei weniger um konkrete weltanschauliche Unterschiede, denn Romney hatte letztlich fast alle orthodoxen Standpunkte des amerikanischen Konservativismus eingenommen. Was Santorum und Gingrich - und vor ihnen die anderen zeitweiligen Favoriten der Tea Party - dem hinzufügten, war lediglich eine Prise

59 Vgl. Jennifer de Pinto / Sarah Dutton, How Mitt Romney Became the Presumptive Nominee, http://www.cbsnews.com/8301-503544_162-57412775-503544/how-mitt-romney-became-thepresumptive-nominee (Abruf am 18. Februar 2013).

60 Vgl. John Sides / Lynn Vavreck, a.a.O. (Fn. 35), S. 46.

61 Vgl. ebenda, S. 42.

62 Vgl. ebenda, S. $46 \mathrm{f}$.

63 Vgl. Richard W. Stevenson, Tea Party Movement Takes the Long View, in: The New York Times (The Caucus - The Politics and Government Blog) vom 9. März 2012, http://thecaucus.blogs. nytimes.com/2012/03/09/tea-party-movement-takes-the-long-view/ (Abruf am 17. Februar 2013).

64 Vgl. Jennifer de Pinto / Sarah Dutton, a.a.O. (Fn. 59).

65 Vgl. ebenda.

66 Vgl. Marty Cohen / David Karol / Hans Noel / John Zaller, The Party Decides. Presidential Nominations Before and After Reform, Chicago 2008. 
zusätzlichen Ressentiments, die Attacke gegen die „liberalen Medien“ und die „versnobten Intellektuellen“, aber kaum ein konkretes Wahlprogramm, das Romney wie einen zu moderaten Kandidaten hätte aussehen lassen. Kurz gesagt: Sie drehten weiter an der Eskalationsspirale populistischer Erregung, benutzten eine Sprache, zu der Romney keinen Zugang hatte - aber das war es dann auch. Entgegen der landläufigen Meinung vertrat ein Großteil der Wähler in den Republikanischen Primaries die Auffassung, dass es sich bei Romney um einen durchaus hinreichend konservativen Kandidaten handelte. Diese Wandlung hatte er, allerdings ohne dabei große Begeisterung auszulösen, am Ende zumindest bewerkstelligt.

Überhaupt sollte sich die Befürchtung, dass ein zu moderater Kandidat vielleicht nicht in der Lage wäre, die Kernwählerschaft der Partei in der Hauptwahl gegen den Präsidenten zu mobilisieren, später als unbegründet erweisen. Romneys Problem bestand vielmehr darin, dass er in einem sich demographisch wandelnden Amerika über diese schrumpfende Kernklientel nicht wesentlich hinausgreifen konnte, vermutlich auch, weil er im Prozess der Vorwahlen weit nach rechts rücken musste. Die „true believers“ der Republikaner aber waren auch 2012 hoch motiviert, denn was diesen Vorwahlkampf wohl noch stärker als sonst überlagerte, war die scharfe Opposition gegen die amtierende Regierung, auch die radikale Ablehnung der Person des Präsidenten. Und weil sich darin alle Republikanischen Wähler so einig waren, spielte das Argument der Wählbarkeit in der Hauptwahl eine überragende Rolle in diesem Vorwahlkampf; und hier besaß Romney entscheidende Vorteile gegenüber seinen Rivalen, die allesamt auf die eine oder andere Weise zu viel Angriffsfläche boten. Selbst die ultrakonservativen Chefideologen der Bewegung wie Ann Coulter und Rush Lambaugh unterstützten daher im Laufe der Primary Mitt Romney, da sie alles dem Ziel unterordneten, einen Wechsel im Weißen Haus herbeizuführen - das Ziel, das bekanntlich in der Hauptwahl am 6. November 2012 verfehlt wurde.

\section{Die US-Präsidentschaftswahl vom 6. November 2012: Obamas Wiederwahl dank verbesserter Zukunftserwartungen, Hurrikan Sandy und einer effektiven Kampagne}

\section{Patrick Horst}

Nachdem Barack Obama im November 2008 mit seiner Wahl zum 44. Präsidenten der USA hohe Erwartungen hinsichtlich einer transformativen Amtsführung geweckt hatte ${ }^{1}$, holte ihn die Realität eines politischen Systems, das auf Gewaltenteilung und Machtkontrolle angelegt ist, schnell wieder ein. ${ }^{2}$ Jedoch gelang es ihm, eine Reihe von bedeutenden Gesetzen und internationalen Verträgen durch den 111. Kongress (2009 bis 2011) zu schleusen. Schon bei der Reform des Gesundheitswesens und der Finanzmarktregulierung musste Obama

1 Vgl. Steven Schier, Obama’s “Big Bang” Presidency, in: The Forum, 8. Jg. (2010), H. 3, Article 13; Theda Skopcol / Lawrence R. Jacobs, Accomplished and Embattled: Understanding Obama's Presidency, in: Political Science Quarterly, 127. Jg. (2012), H. 1, S. 1 - 24.

2 Hierzu jetzt Christoph M. Haas / Wolfgang Jäger (Hrsg.), What a President Can - Barack Obama und Reformpolitik im Systemkorsett der USA, Baden-Baden 2012. 